\title{
The Design and Implementation of Photoelectric Sensor for Yarn Color Fault Detection
}

\author{
http://dx.doi.org/10.3991/ijoe.v12i02.5035 \\ SHENG Fenhua ${ }^{1}$, CHEN Zujue ${ }^{2}$ \\ ${ }^{1}$ Suzhou college of Information Technology, China; \\ ${ }^{2}$ Jiangxi University, China
}

\begin{abstract}
The paper mainly aimed at solving the problem of yarn color fault detection. Yarn with different color is hard to detect in yarn production, a special photoelectric sensor is designed in this paper. First, this paper analyzed the requirement of light source and photoelectric receiver in the photoelectric sensor, and designs the light path and driver circuit. Then this paper analyzed the amplifier circuit and noise in the photoelectric sensor, with an amplifier circuit of minimal noise proposed at last. Finally, this paper tested the yarn color fault detection system with virtual instrument, and the test results showed a great application prospect of the photoelectric sensor. Photoelectric yarn clearer was the first type of electronic yarn clearer, but due to the under development of the optical technology and measurement technology, the photoelectric yarn cleaner can't meet the requirements of textile production, gradually replaced by capacitive yarn cleaner. Though photoelectric yarn cleaner had a good visual conformity degree, it's still a unreplaceable method in colored yarn faults [1].
\end{abstract}

Index Terms-Photoelectric Sensor, Yarn, Color, Fault Detection

\section{INTRODUCTION}

\section{A. Design of Photoelectric Sensor}

This paper designed a photoelectric sensor module to solve the problem. The module is shown in Figure 1.

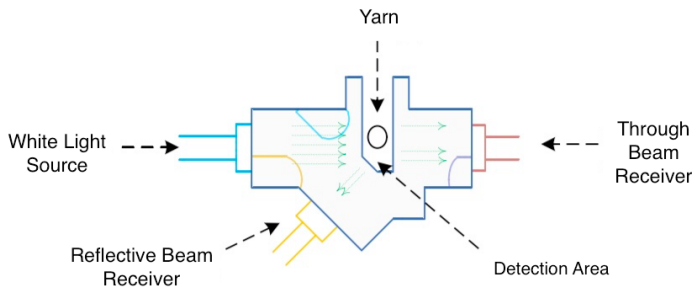

Figure 1. The photoelectric sensor module

Photoelectric sensor consists with the light source, reflective beam receiver and through beam receiver $[2,3]$. When the light source emits light on the yarn, some will be absorbed and some will reflect. The reflective beam not only contains the yarn's color (gray) information, but also the yarn's diameter information. The reflective beam received by reflective beam receiver. When the color or diameter of the yarn changes, the reflective beam's luminous intensity will also change. In order to get the color information, the diameter information must be removed, so the through beam receiver is added. The change in through beam receiver is depending on the diameter change of the yarn. Parts of the light come from the light source will be blocked by the yarn, the left parts will received by the through beam receiver. The larger the yarn's diameter, the lesser luminous intensity the through beam receiver will receive. The through beam receiver aim to get the yarn's diameter information, in a consequence of dismiss relationship of reflective beam and the yarn's diameter information.

The components selection and optical path design of the photoelectric sensor is described below.

\section{B. Light Source}

Due to the restriction of electronic yarn cleaner's size, the light source should be as small as possible, while the light source keep a high brightness. The color information detect from the yarn not only related to the reflective beam, also has a relationship to the spectrum of light source. This paper aim to detect the yarn's color fault among the visible spectrum, thus the light source must contain the entire visible spectrum. To sum up, this paper chooses the high brightness white LED as light source of photoelectric sensor.

This white LED used in this paper is made of Indium gallium nitride ( $\mathrm{InGaN}$ ) chips and yttrium aluminum garnet (YAG) phosphor. The peak of InGaN chip is $465 \mathrm{~nm}$ blue light, this blue light excite the YAG phosphor to emit yellow light peak at $550 \mathrm{~nm}$. Parts of the blue light emit from the chip absorbed by the YAG phosphor, the left parts mixed with the yellow light become the white light.

The spectrum of the whit light is shown in Figure 2, among the visible spectrum, the white light's brightness of single LED is above $1 \mathrm{~cd}$, with color temperature of $6500 \mathrm{k}$, and a rendering index greater than 70 . The white light LED has the quality of stability and high brightness, suitable for the detection of yarn color faults.

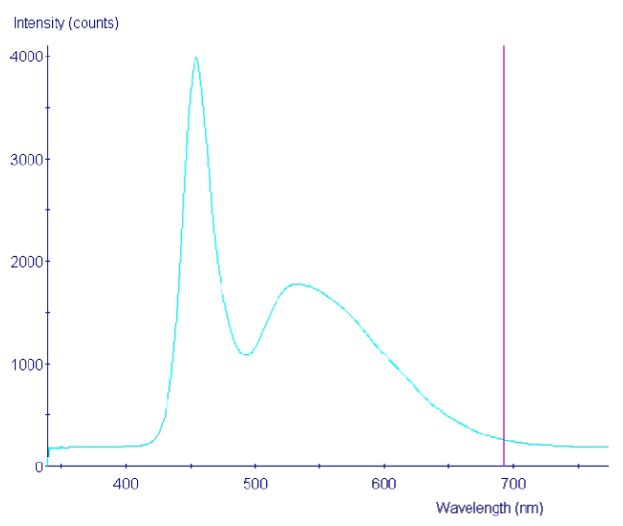

Figure 2. Spectrum of the White LED 


\section{Photoelectric Receiver}

There are a lot of kinds of photoelectric detection device, such as: phototube, photomultiplier tubes, photocell, photoelectric diode, and photoelectric triode, etc.

In this paper, due to the restriction of the photoelectric device's size, some of the devices can't be selected, such as phototube, photomultiplier tubes, photo cell. Also the speed of the yarn can get up to $20 \mathrm{~m} / \mathrm{s}$, thus the photoelectric detection device must have a good dynamic characteristics. To sum up, this paper chooses the diode as the photo electric receiver. The diode also has the advantages of stability, high sensitivity and low price.

This paper selects the photoelectric diode manufactured by Beijing's north optoelectronic co., LTD, model $2 \mathrm{cu} 2 \mathrm{e}$. The diode has $400 \mathrm{~nm}$ to $1100 \mathrm{~nm}$ spectral range, peak at $800 \mathrm{~nm}$, maximum working reverse voltage of $50 \mathrm{v}$, maximum dark current less than $0.1 \mathrm{uA}$. The diode under $1000 \mathrm{Lx}$ intensity, 2856k junction temperature light, has the light current up to $40 \mathrm{uA}$, and the response time is higher than 200ns. The characteristic of the diode described above fit the requirements of this paper.

\section{Light Path}

The detection theory of the photoelectric sensor is shown in Figure 3.

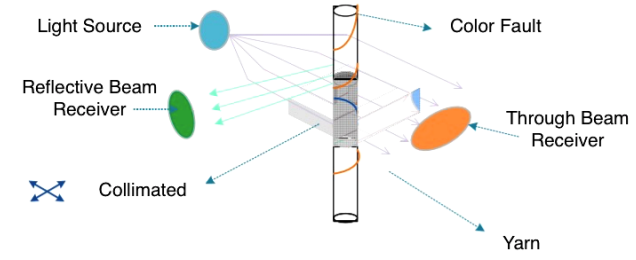

Figure 3. Detection Theory of Photo electronic sensor

As shown in figure 3, when the yarn go through the detection area of the photo electronic sensor, the reflective beam receiver and through beam receiver will both receive a flare which will change with the yarn's diameter and color, the receiver will get the information of the yarn's diameter and color.

The method described above require the light to be collimated, and at same intensity. LED is a point light source, the light emit from it in radiation at certain angle. To make the light parallel to each other, the collimation light path is added to the photo electronic sensor, shown in Figure 4.

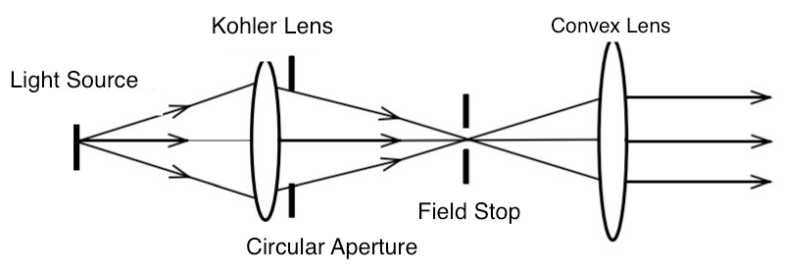

Figure 4. LED collimation light path

LED collimation light path use the method of Kohler illumination to get uniform parallel light. Kohler lens zoom up the light emit from the light source, put light in front of the convex lens' focal plane, and the field stop also at the convex lens' focal plane, the convex lens imaging the light in infinite distance. The aperture in the light path determine the aperture angle of the system, also sets the resolution and contrast. Kohler illumination can generate parallel light, make the measured intensity of illumination uniformity, this process make sure all parts of the yarn can be detected and identified. This paper select the $4 \mathrm{~mm}$ Kohler lens, with focal length of $2 \mathrm{~mm}$, the convex lens' diameter is $4 \mathrm{~mm}$ with $2 \mathrm{~mm}$ focal length.

Meanwhile, in the yarn fault detection, the diode must receive as much as light as possible, for all the light goes to the receiver must cover the diode's photosensitive surface as much as possible, so a condenser lens is added before the diode, shown in Figure 5. The condenser lens has a diameter of $5 \mathrm{~mm}$, a focal length of $2 \mathrm{~mm}$.

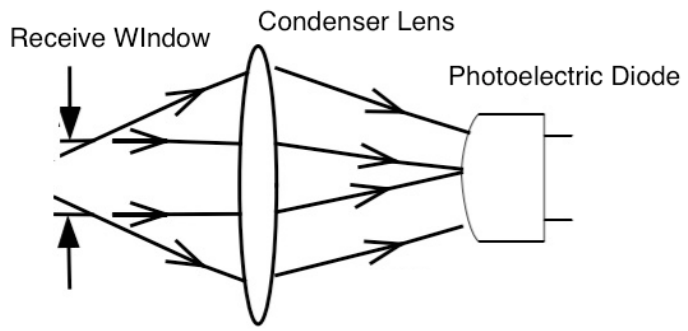

Figure 5. The Condenser Light Path of Photoelectric Diode

\section{Design of The Light Source Driving Circuit And SIGNAL PROCESSING CIRCUIT}

The method of photoelectric yarn flaw detection belongs to the weak light detection, light intensity change caused by yarn fault is very small, thus its requirements on the stability of light source is very rigid. In addition to materials of LED itself, the light source's luminous stability is mainly decided by the LED light driver circuit. In this paper, after considering various LED light driver circuit, decide to adopt the LED constant current driver circuit. Driver circuit is shown in Figure 6

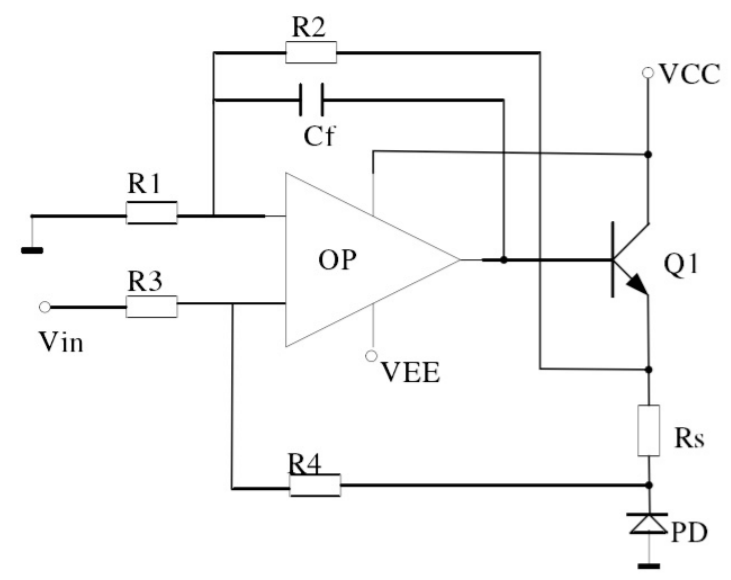

Figure 6. Constant Current Driver Circuit.

This circuit uses the voltage controlled current source, which is a improved Howland current pump, and it can provide photoelectric diode with constant drive current. Compared with the switching PWM circuit, this circuit has a better linearity, which can effectively reduce the drive current ripple. Output current $I_{\text {out }}$ is controlled by Input voltage $V_{i n}$, the input voltage is generated by sys- 
tem chip, the relationship between output current $I_{\text {out }}$ and input voltage $V_{\text {in }}$ is:

$$
I_{\text {out }}=V_{\text {in }} \frac{R_{4}}{R_{3} \times R_{s}}
$$

Which $R_{1}=R_{3}, R_{2}=R_{4}$.

In the circuit, select OPA350 of TI company as op-amp. OPA350 is R - R CMOS' operational amplifier, the bandwidth is up to $38 \mathrm{MHZ}$, pressure swing rate is 22 v/us, the op-amp has a high switching speed, low noise. In order to meet the circuit requirements,

$$
\begin{aligned}
& \text { Set } R_{3}=100 \mathrm{~K} \Omega, R_{4}=10 \mathrm{~K} \Omega, R_{s}=10 \Omega, \text { form } \\
& \text { equation } 1, I_{\text {out }}=V_{\text {in }} / 100 \text {, set } I_{\text {out }}=30 \mathrm{~mA} \text {, then } \\
& V_{\text {in }}=3 \mathrm{~V} \text {. The simulation result is shown in Figure } 7 .
\end{aligned}
$$

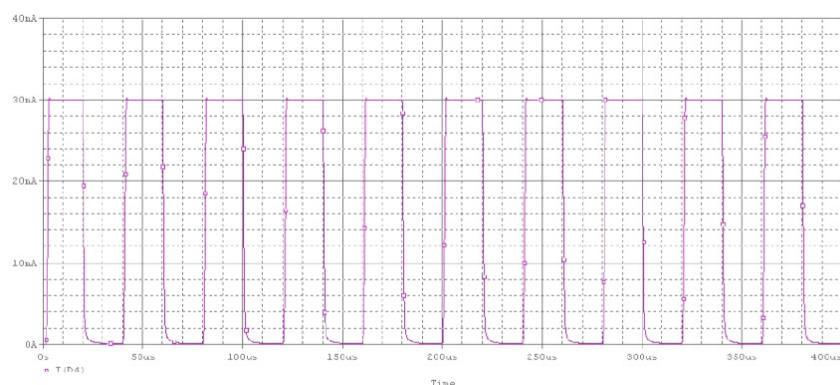

Figure 7. Constant Current Driver Circuit Simulation Result

\section{TRANSRESISTANCE AMPLIFIER}

The detection of the photoelectric current of the photoelectric diode is crucial to the signal processing. Because of the photoelectric current signal is very weak, the design of the pre-amplifier is particularly important. Those should be considered, such as noise and sensitivity and response time. The noise affects the detection of the photoelectric current most.

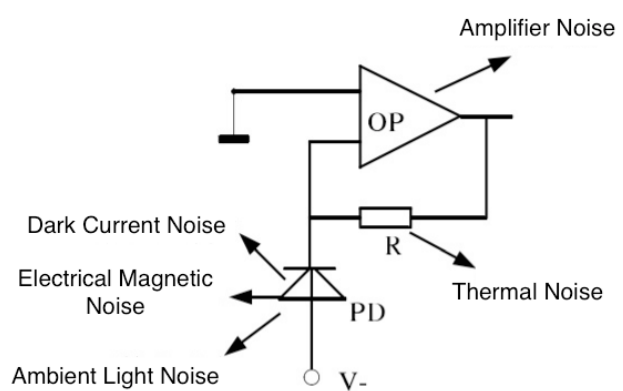

Figure 8. Noise in the Photoelectric Signal Amplifying Circuit

Photoelectric signal is very weak, in the process of transmission, detection, and amplification, it will always be interfered by various inevitable factors. The noise introduced in the process of photoelectric signal amplifying is shown in the figure 8 . The quantum noise from the surrounding space and environment, the electrical magnetic interference from the surrounding electronic equipment can be prevented through such as shielding and grounding. The ambient light interference can be eliminated through compensation. The internal noise, such as dark current noise, thermal noise, amplifier noise, can' t completely eliminated but only can reduce them as much as possible through circuit design[4].

The amplifier can amplify the signal as much as possible, but at the same time, the noise is also being amplified, if the detected photoelectric signal is too weak, is likely to be covered up by the noise.

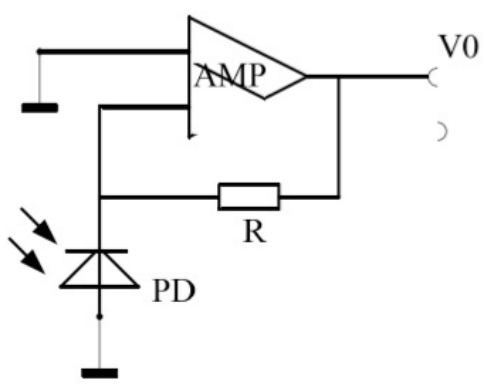

Figure 9. Transresistance Amplifier

Response time refers to the time of circuit from receiving a mutation signal to a stable state. This paper adopted the LED drive signal of $25 \mathrm{KHz}$ square wave modulation signal, require a fast response speed of the circuit.

This paper use transresistance amplifiers to amplify the primary signal of the photoelectric diode. The structure of the transresistance amplifyer is shown in Figure 9. Transresistance amplifiers use a resistor with high resistance as feedback, has the characteristic of high gain and high impedance, at the same time, the transresistance has low noise, large bandwidth and wide dynamic range, commonly used in circuit with requirements of speed.

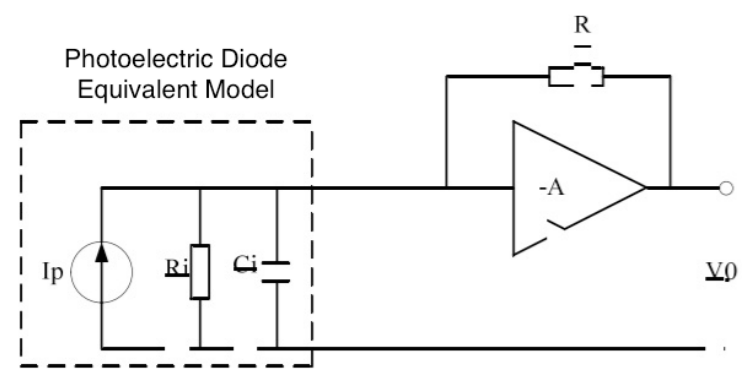

Figure 10. Transresistance Amplifier Equivalent Model

As shown in figure 10 is the transresistance amplifiers equivalent model, the photoelectric diode can be equivalent to a constant current source $I_{p}$, with paralleled connection of a large resistance $R_{i}$ and a small capacitance $C_{i}$. In the circuit, $R_{i}, C_{i}$ respectively, as the amplifier's input resistance and input capacitance. Set operational amplifier as ideal, $\mathrm{R}$ as the feedback resistance, then the transfer function of the circuit is:

$$
H_{1} \omega=\frac{V_{0}(\omega)}{I_{p}(\omega)}=\frac{-A}{\frac{1+A}{R}+\frac{1}{R_{i}}+j \omega C_{i}}
$$


In general intermediate frequency of operational amplifier gain $A\rangle\rangle 1$, and the equivalent resistance of photoelectric diode $\left.\left.R_{i}\right\rangle\right\rangle 1$, then, equation (2) is:

$$
H_{1} \omega=\frac{V_{0}(\omega)}{I_{p}(\omega)}=\frac{-A}{\frac{A}{R}+j \omega C_{i}}=-\frac{R}{1+\frac{j \omega R C_{i}}{A}}
$$

The transresistance' $\mathrm{s}$ bandwidth is similar to:

$$
\omega_{0}=\frac{A}{R C_{i}}
$$

If $R \rightarrow 0$, then the operational amplifier tend to be more open. Transfer function for this time is:

$$
H_{1} \omega=\frac{-A}{\frac{A}{R_{i}}+j \omega C_{i}}=-\frac{A R_{i}}{1+j \omega R_{i} C_{i}}
$$

The bandwidth is:

$$
\omega_{1}=\frac{1}{R C_{i}}
$$

From equation (4) and (6), the $\left.\left.\omega_{0}\right\rangle\right\rangle \omega_{1}$, meaning the transimpedance amplifier bandwidth is bigger than no feedback amplifier bandwidth, compared with a high impedance amplifier, transimpedance amplifier bandwidth broadening A times, and is proportional to the amplifier' $\mathrm{s}$ gain, and is inversely proportional to the feedback resistance. Reduce $C_{i}$ can increase the bandwidth of the amplifier, lower the noise. And by reducing $C_{i}$, the response speed of the photoelectric senor also improved.

To sum up the above analysis, this paper adopts the circuit as below:

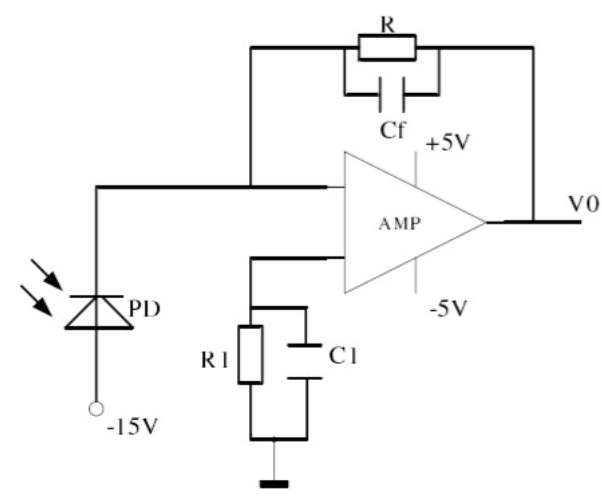

Figure 11. Adopted Transresistance Amplifier Circuit

The operational amplifier chose the precision low noise op-amp AD8616 of AD company. The bandwidth product is up to $24 \mathrm{MHz}$. Pressure swing at a rate of $12 \mathrm{~V} / \mu \mathrm{s}$, which can guarantee a wide response speed; the input bias current is $1 \mathrm{pA}$, which can effectively reduce the noise from the op-amp. $R_{1}$ and $C_{1}$ are resistance and capaci- tance compensations. $C_{f}$ is feedback capacitance, used to offset the photoelectric diode junction capacitance influence on subsequent circuit, and prevent the amplifying circuit self-excited vibration oscillation, $R$ and $C_{f}$ are decider in transresistance amplifiers in the circuit performance, they will affect the frequency stability and the noise performance of detection circuit. In practical applications, the $R=R_{1}=1 M \Omega, C_{1}=C_{f}=1.5 p F$.

\section{SignAl ACQUiSITION AND PROCESSING BASED ON VIRTUAL INSTRUMENT}

Base on the design progress and analysis above, the yarn fault detection system mainly want to collect data of $25 \mathrm{KHz}$ square wave, and the NI company' s PCI-6251 high precision data acquisition board is well fit to our purpose[5]. And with NI-DAQmx driver and Measurement \&Automation Explorer (MAX) software, combined with Labview, the acquisition data of reflective beam information and through beam information will save to .xcl file after the experiment.

The experiment set up with two different diameter of uniform white yarn production as yarn gauge, roving line diameter is about $0.12 \mathrm{~mm}$, spinning line diameter about $0.08 \mathrm{~mm}$ in diameter. Dye the yarn of different thickness separately with green, yellow, black and orange, as different yarn color faults. Testing for each color, save the tests data, then use Matlab to de-noising. Due to the limitation of experimental conditions, this paper only conduct the experiment to test the system' s static performance. The results is shown in Figure 12 and 13 in below.

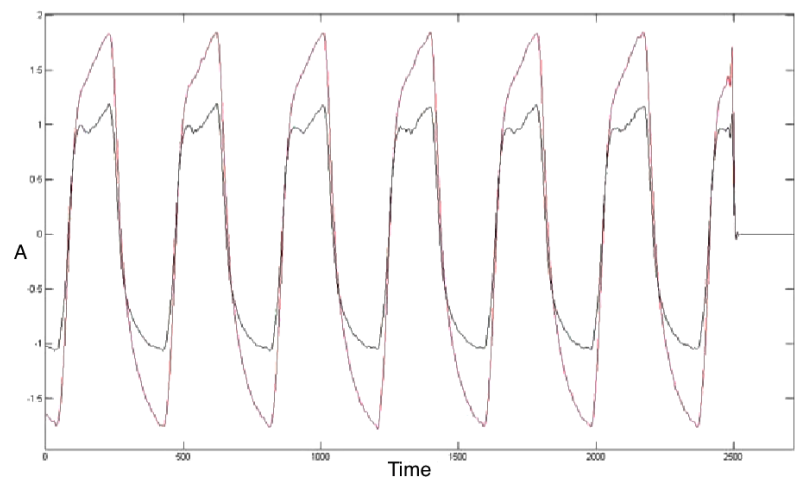

Figure 12. Different diameter of yarn with red color fault

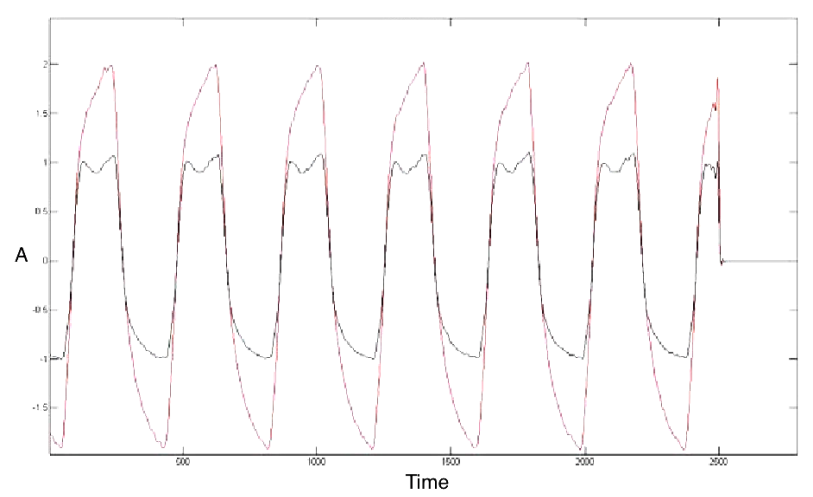

Figure 13. Different diameter of yarn with black color fault 
PAPER

The Design and IMPlementation of PhotoeleCtric SEnsor For Yarn Color Fault Detection

As shown in Figure 12 to 13 , different thicknesses on red yarn defects and black yarn yarn defects signal in contrast, signal is larger for the test results of roving line, small signal for the test results of spinning line, yarn diameter changes has a clear impact on the detection signal, the larger the diameter of the yarn, the more obvious effect on light reflection.

\section{CONCLUSION}

The experimental results show that the design of photoelectric sensor can be effectively detected yarn of different color fault. Results also show that the reflection ending of the photoelectric sensor at the same time, in addition to the different color yarn defects on the yarn can be effectively detect, its detection signal is sensitive to changes in yarn diameter. In the practical work, we hope that reflex photoelectric sensor output signal at the receiving end is only related to the different color yarn faults, not the diameter and the relevant department. So in the design of photoelectric sensor, we joined the photoelectric transmission and the receiving end, to measure the diameter of the yarn information as a compensation signal due to the yarn diameter changes effect the output signal. The virtual instrument experiment result testifies that the photoelectric sensor and the method proposed in this paper to detect yarn fault is both valid and efficient.

\section{REFERENCES}

[1] Yadav, V. K., Joshi, S. D., Ishtiaque, S. M., \& Chatterjee, J. K. 2014. Yarn fault classification: a signal processing approach using multiple projections. Journal of the Textile Institute, 1-9.

[2] Gonçalves, N., Carvalho, V., Belsley, M., Vasconcelos, R. M., Soares, F. O., \& Machado, J. 2015. Yarn features extraction using image processing and computer vision-A study with cotton and polyester yarns. Measurement, 68, 1-15. http://dx.doi.org/10.1016/ j.measurement.2015.02.010

[3] Sengupta, A., Roy, S., \& Sengupta, S. 2015. Development of a low cost yarn parameterisation unit by image processing. Measurement, 59, 96-109. http://dx.doi.org/10.1016/j.mea surement.2014.09.028

[4] Ruirui, Z., \& Xiao, G. 2012. The Application and Development of Photoelectric Sensor. Energy Procedia, 17, 1304-1308. http://dx.doi.org/10.1016/j.egypro.2012.02.243

[5] Zukovic, S., Medenica, M., Draganic, A., Orovic, I., \& Stankovic, S. 2014. A Virtual Instrument for Compressive Sensing of multimedia signals. In ELMAR (ELMAR), 2014 56th International Symposium (pp. 1-4). IEEE.. http://dx.doi.org/10.1109/elmar. 2014.6923339

\section{AUTHORS}

SHENG Fenhua is with Suzhou college of Information Technology, Wujiang Suzhou, Jiangsu, CO215200, China

CHEN Zujue is with School of Computer Science and Telecommunication Engineering, Jiangsu University, Zhenjiang, Jiangsu, CO212000, China

Submitted 17 September 2015. Published as resubmitted by the authors 13 January 2015. 